\title{
Self-assembled Supramolecular Gels of Reverse Poloxamers and Cyclodextrins
}

\author{
Eneko Larrañeta, José Ramón Isasi* \\ Departamento de Química y Edafología, Facultad de Ciencias, \\ Universidad de Navarra, 31080 Pamplona, Navarra, Spain
}

This document is the unedited Author's version of a Submitted Work that was subsequently accepted for publication in Langmuir, copyright (C) American Chemical Society after peer review. To access the final edited and published work see http://pubs.acs.org/doi/abs/10.1021/la3024452

* Corresponding author. Tel.: +34-948-425600; fax: +34-948-425740.

E-mail address: jrisasi@unav.es (J.R. Isasi). 
ABSTRACT: A series of supramolecular aggregates were prepared using a poly(propylene oxide)-poly(ethylene oxide)-poly(propylene oxide) (PPO-PEO-PPO) block copolymer and $\beta$ - or $\alpha$-cyclodextrins (CD). The combination of $\beta$-CD and the copolymer yields inclusion complexes (IC) with polypseudorotaxane structures. These are formed by complexation of the PPO blocks with $\beta$-CD molecules producing a powder precipitate with a certain crystallinity degree that can be evaluated by X-ray diffraction (XRD). In contrast, when combining $\alpha-C D$ with the block copolymer, the observed effect is an increase in the viscosity of the mixtures yielding fluid gels. Two cooperative effects come into play: the complexation of PEO blocks with $\alpha-\mathrm{CD}$ and the hydrophobic interactions between PPO blocks in aqueous media. These two combined interactions lead to the formation of a macromolecular network. The resulting fluid gels were characterized using different techniques such as differential scanning calorimetry (DSC), viscometry and XRD measurements.

\section{Introduction}

The supramolecular self-assembled aggregates have attracted much attention over the past decades, especially with respect to their feasible pharmaceutical and biological applications for some purposes such as gene carriers or drug delivery systems ${ }^{1-8}$. These structures could possess the same properties of covalent crosslinked hydrogels adding new promising features such as a quick degradation process ${ }^{3}$, stimuli responsive properties $^{2}$ or the capability of gelling "in situ" by physical crosslinking between different molecules or subunits ${ }^{1,3,5,8}$.

A group of molecules frequently used to prepare self-assembled systems are cyclodextrins (CD), which form inclusion complexes with different molecules, 
including polymers ${ }^{9}$. Some previous works have reported the formation of selfassembled gels between two polymers: one with pendant cyclodextrin moieties and the other with a complementary substituent that fit into the CD cavities yielding inclusion complexes $^{4,7}$.

Among the molecules that produce stable inclusion complexes with cyclodextrins, linear polymers such as polyethylene oxide (PEO) and polypropylene oxide (PPO) generate polyrotaxane type structures (Fig. 1a) ${ }^{3,5,10}$. Cyclodextrins yield inclusion complexes also with $\mathrm{PEO} / \mathrm{PPO}$ block copolymers yielding selective polyrotaxanes depending on the type of CD used ${ }^{11-14} \cdot \beta$ - and $\gamma$-CD form complexes with PPO blocks ${ }^{14}$ ${ }^{15}$ and $\alpha-C D$ interacts with PEO blocks ${ }^{16,17}$. PPO blocks do not fit into the hydrophobic cavity of $\alpha-C D$, which is smaller than those of $\beta$ - and $\gamma$-CDs.

Amphiphilic PEO/PPO block copolymers, synthesized and distributed by BASF under the name of Pluronic and also known as poloxamers, form micelles or other selfassembled structures in water under certain conditions ${ }^{18,19}$. The reverse poloxamers (Pluronic R) are also PEO/PPO linear triblock copolymers, but the configuration of the blocks is changed: the outer hydrophobic blocks are PPO, with a central hydrophilic block made of EO units (Fig. 1b). Although the PPO chain is too large to penetrate the cavity of $\alpha-\mathrm{CD}$, it has been proved that the $\alpha$-cyclodextrin molecules can pass over PPO blocks in order to form inclusion complexes with the central PEO block ${ }^{13,20}$. 
(a)

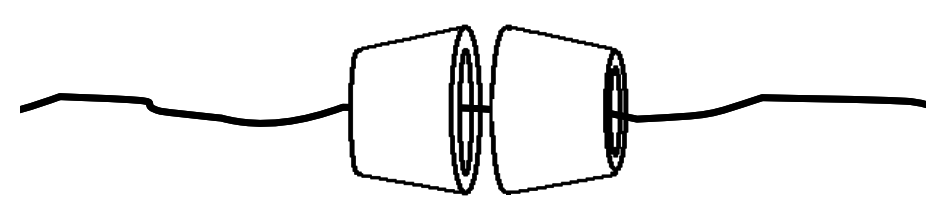

(b)

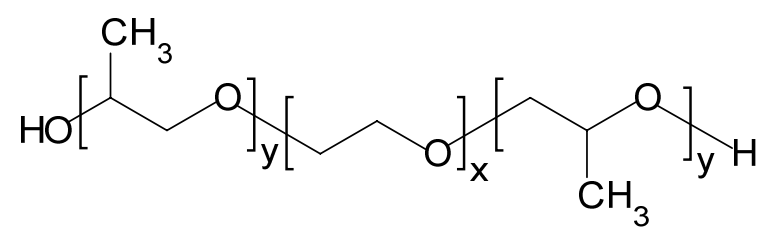

Figure 1. Scheme of a polyrotaxane complex between PEO and two $\alpha$-CD molecules (a) and chemical structure of a reverse poloxamer (Pluronic R) (b)

Direct poloxamers with different block lengths have been combined with cyclodextrins to yield different types of products, either solid precipitates or gels. It has been reported that mixtures of PEO/PPO copolymers and $\alpha-C D$ can form gels ${ }^{5}$. Besides the associations between the polyrotaxane blocks of complexed PEO molecules, there is also a hydrophobic interaction between the PPO blocks (Figure 2a). In contrast, for poloxamer/ $\beta-\mathrm{CD}$ mixtures, there are not hydrophobic interactions: PPO blocks are complexed with $\beta-\mathrm{CD}$ and these polyrotaxane structures are responsible for the aggregation process (Figure 2b). These associations lead to the formation of a white precipitate with a certain crystalline structure ${ }^{13,14}$. The interactions between the blocks are easily broken when the gels or the solid complexes come in contact with a large amount of water. This can be very useful for some applications such as drug release resulting from a complete erosion of the matrix. 
(a)

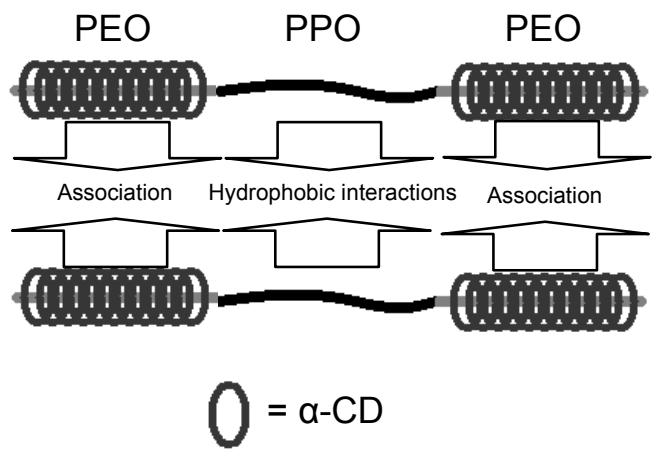

(b)

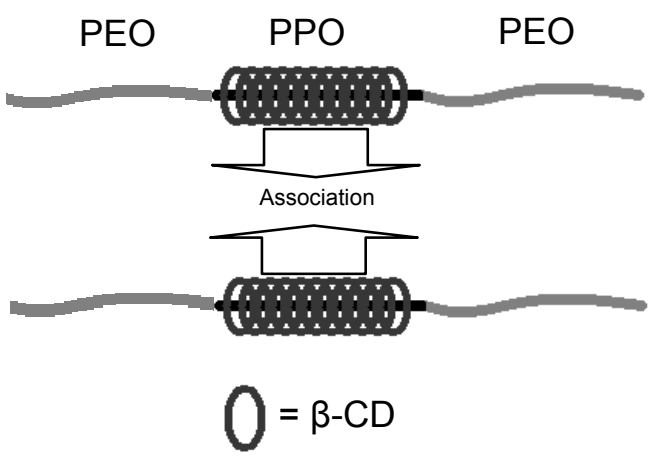

Figure 2. Structures of the complexes between Pluronic copolymers and $\alpha$ - (a) and $\beta$-CD (b).

Recently, some studies have demonstrated the self-organization of Pluronic/natural $\beta$ CD systems in crystalline domains ${ }^{21-24}$. These studies prove that a complex with a certain crystalline order is obtained when combining PEO-PPO-PEO block copolymers with $\beta$-CD. As pointed above, $\beta$-CD forms inclusion complexes with the PPO blocks, and these complexed blocks tend to aggregate. These inclusion compounds exhibit a peculiar crystal morphology, evaluated by X-ray diffraction and electronic microscopy, where the central PPO polyrotaxane structures self-assemble yielding a layer with the PEO segments protruding from both sides of these nanoplatelets ${ }^{21,24}$. Li et al. ${ }^{5,10,20,25}$ have studied inclusion complexes between $\alpha-C D$ and poly(ethylene oxide) and its copolymers including Pluronics. In a recent work, they used several reverse poloxamers with varying molecular weights in order to form inclusion complexes with $\alpha-C D$. These rotaxane type structures are formed by mixing $\alpha-\mathrm{CD}$ and copolymer solutions. In a short amount of time, the solutions become turbid forming a precipitate that can be isolated. Mixtures of PEO-PPO-PEO copolymers (i.e. direct poloxamers) and $\alpha$-CDs have been also studied ${ }^{2,5}$. In this case, both precipitates and gels have been obtained depending on the PEO/ $\alpha$-CD ratio used. In the case of PPO polyrotaxanes, Harada et al. also studied the complexation of poly(propylene oxide) with $\beta$ - and $\gamma-\mathrm{CD}^{14}$. 
In this work, we have studied the capability of Pluronic 10R5 $\left(\mathrm{PPO}_{8} \mathrm{PEO}_{22} \mathrm{PPO}_{8}\right)$ to form either gels or precipitates when mixed with $\alpha-$ or $\beta-C D$ in certain ratios. We have also compared the behaviour of natural and modified CDs in order to elucidate the mechanisms of the self-assembling process. Different techniques were used to characterize the resulting complexes: X-ray diffraction (XRD), nuclear magnetic resonance (NMR), differential scanning calorimetry (DSC) and viscosity measurements.

\section{Experimental methods}

Materials. Pluronic 10R5 $\left(\mathrm{PPO}_{8} \mathrm{PEO}_{22} \mathrm{PPO}_{8}\right)$ is a viscous $\left(440 \mathrm{cP}\right.$ at $\left.25^{\circ} \mathrm{C}\right)$ colourless liquid. The Pluronic 10R5 sample was kindly donated by BASF and it was used as received. The molecular weight $\left(\mathrm{M}_{\mathrm{w}}=1950 \mathrm{~g} / \mathrm{mol}\right)$ and chemical composition given by the manufacturer were corroborated by ${ }^{1} \mathrm{H}$ NMR (Bruker DPX 300 at $298 \mathrm{~K}$ ). Both natural cyclodextrins, $\beta$ - and $\alpha-\mathrm{CD}$, were obtained from Wacker Chemie AG and were used without further purifications. Hydroxypropyl- $\alpha-C D$ was from Fluka and hydroxypropyl- $\beta-\mathrm{CD}$ was obtained from Cyclolab. D-(+)-glucose was from Sigma.

Preparation of complexes. All complexes were formed by mixing a determinate amount of 10R5 with an aqueous solution of $\mathrm{CD}$ by vigorous stirring. Prior to any measurements the resulting mixtures were kept at room temperature for at least one night.

For the $\alpha$-CD complexes, the viscous fluids obtained were homogeneous and stable and were analysed without purification. In the case of the $\beta$-CD complexes, a white powder appears at the bottom of the flask. The supernatant was carefully removed and the 
powder was freeze-dried and washed with tetrahydrofuran (THF) in order to remove the excess of uncomplexed poloxamer ${ }^{14}$. Yield diagrams were performed for $\beta$ $\mathrm{CD} /$ poloxamer complexes in order to check the stoichiometry of the complexes. The resulting complexes were weighed after the purification process.

NMR measurements. The solid-state ${ }^{13} \mathrm{C} \mathrm{CP/MAS} \mathrm{NMR} \mathrm{were} \mathrm{measured} \mathrm{on} \mathrm{a} \mathrm{Bruker}$ AV-400 NMR WB with a sample spinning rate of $12 \mathrm{kHz}$ at room temperature. $\mathrm{CP}$ spectra were acquired with a $4 \mu$ s proton $90^{\circ}$ pulse, a $2.5 \mathrm{~ms}$ contact time, and a $5 \mathrm{~s}$ repetition time. (The poloxamer methyl signal was used to calculate the stoichiometries of the complexes.)

Elemental analysis measurements. The elemental analysis were carried out with a Leco CHN 900 using about $1 \mathrm{mg}$ of sample.

Wide-angle X-ray diffraction measurements. The diffractograms of $\beta$-CD complexes and $\alpha$-CD gels were carried out in a Brüker D8 Advance X ray diffractometer equipped with a $\mathrm{X}$ ray generator, Kristalloflex $\mathrm{K} 760$, using the radiation $\mathrm{K}_{\alpha 1}$ of the $\mathrm{Cu}(\lambda=1,5417$ $\AA$ ), and a scanning speed of $0.4^{\circ}$ per minute. $\alpha$-CD/10R5 hydrogels were vacuum dried at $60^{\circ} \mathrm{C}$ overnight before the measurements.

Phase diagrams measurements. Different $\alpha-C D / 10 R 5$ gel samples were prepared in $8 \mathrm{~mL}$ flasks varying the $\alpha-\mathrm{CD} /$ copolymer ratio. The samples were placed in a water bath equipped with a thermostatic head. The state of the samples was evaluated for each temperature by direct observation. This state was characterized as "gel" if the mixture did not flow when the flask was kept inverted during at least $10 \mathrm{~s}$, and the state was characterized as "sol" if it flows in that time interval. Glass vials of $23 \mathrm{~mm}$ diameter and $5 \mathrm{~g}$ samples were used for this analysis. The influence of experimental conditions on the 
result of this procedure is discussed in the following section. The temperature ranged between 10 and $60^{\circ} \mathrm{C}\left( \pm 0.1^{\circ} \mathrm{C}\right)$, using $5^{\circ} \mathrm{C}$ steps; a stabilization time of 10 minutes was considered as an appropriate delay time prior to measuring at each temperature.

Viscosity measurements. The viscosity of the $\alpha-C D /$ copolymer hydrogels was evaluated using a Haake Viscotester 550 rotational viscometer with an NV rotor and equipped with a thermostatic bath Thermo Phoenix II. All measurements were performed at $25^{\circ} \mathrm{C}$ and the range of shear rates covered was between 5 and $40 \mathrm{~s}^{-1}$. After a stabilization time of 30 seconds, an average of 100 data points was recorded during a measuring period of 60 seconds. Because of the thixotropic behaviour of the gels, it is important to use a short stabilization time and a constant measuring period for all the samples. For these measurements, no significant changes in the viscosities were detected.

DSC analysis. The thermal analysis of gels was performed using a DSC (differential scanning calorimeter) TA Instruments (DSC 2920) calibrated with indium. The samples were kept at $140^{\circ} \mathrm{C}$ for 20 min to remove the excess water. Two scans were registered between $-100^{\circ} \mathrm{C}$ and $140^{\circ} \mathrm{C}$ using a scan speed of $20^{\circ} \mathrm{C} / \mathrm{min}$. Glass transition temperatures were calculated as the half-height of the corresponding heat-capacity jump.

\section{Results and discussion}

Complex formation of 10R5 with $\boldsymbol{\beta}$-CD. When Pluronic $10 \mathrm{R} 5$ is mixed with $\beta-C D$ solutions a precipitate appears, in a few minutes, at the bottom of the flask (see Supporting Information, Figure S1). Although these mixtures initially present a 
colloidal aspect, coalescence processes yield a white precipitate that settles down. According to the literature, inclusion complexes between $\beta-\mathrm{CD}$ and polypropylene oxide (PPO) can be obtained ${ }^{11,14}$. In the case of reverse poloxamers, the interaction must be produced by the selective formation of complexes between $\beta-\mathrm{CD}$ and the PPO blocks of these copolymers. These complexes are supposed to have a polypseudorotaxane type structure, as occurs between $\beta-\mathrm{CD}$ and PPO. When the cyclodextrin molecules form inclusion complexes with the PPO blocks these structures tend to associate between them (Fig. 2) and, in the end, the aggregates yield a precipitate. The kinetics of this process depends on several factors such as the $\mathrm{CD} /$ copolymer ratio or the total solute concentration. For high $\mathrm{CD} /$ copolymer ratios, the cloudiness appears immediately and the coalescence process takes hours; in other cases, the whole process can last days (see for instance Figure S2 in Supporting Information). Varying the copolymer/ $\beta-C D$ ratio, the amount of obtained complex changes. Figure 3 shows the variation of the yield of complex formation with the propyleneoxyde $(\mathrm{PO}) / \beta$ $\mathrm{CD}$ mole ratio. The graph shows a maximum at a $\mathrm{PO} / \mathrm{CD}$ ratio at about 2 . According to the size of the $\beta-C D$, two PO units can fit inside its cavity. Therefore, the complex has a defined stoichiometry of two PO units per $\beta$-CD molecule. This is consistent with the results found by Harada et al. ${ }^{14}$. 


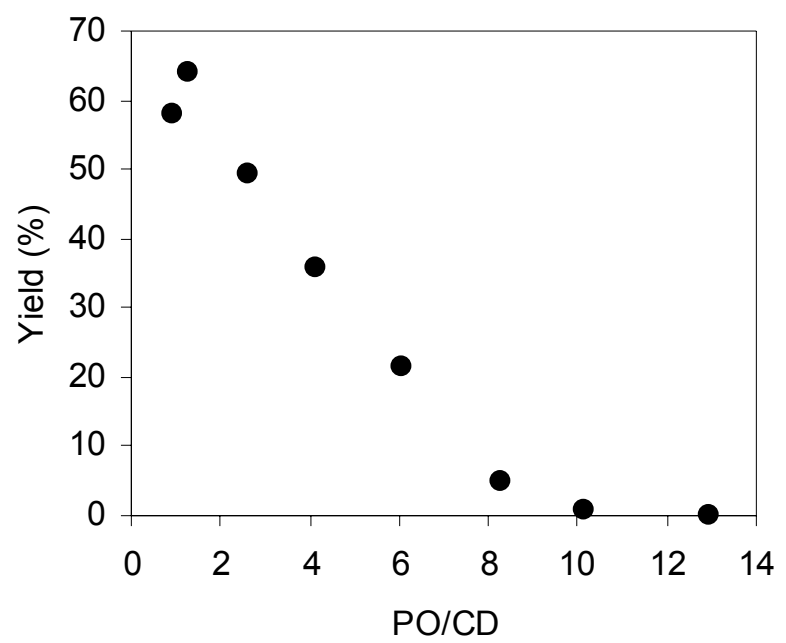

Figure 3. Formation yields for the complexes between $10 \mathrm{R} 5$ and $\beta-\mathrm{CD}$ as a function of the initial $\mathrm{PO} / \beta-\mathrm{CD}$ mole ratio.

CP/MAS NMR measurements and elemental analysis were carried out in order to confirm this stoichiometry results (Fig. 3). Two samples were prepared from 2\% aqueous mixtures, one using the $\mathrm{PO} / \mathrm{CD}$ mole ratio of the maximum in Figure 3 (i.e. 2), and the other one using a higher $\mathrm{PO} / \mathrm{CD}$ mole ratio (ca. 4). The $\mathrm{PO} / \mathrm{CD}$ mole ratios calculated by NMR and elemental analysis in the final solid complexes are shown in Table 1. Thus, the stoichiometry obtained in the yield graph, 2 PO units per $\beta$-CD molecule, is consistent with that calculated from these results.

Table 1. NMR and elemental analysis calculated PO/CD mole ratios for $10 \mathrm{R} 5 / \beta-\mathrm{CD}$ complexes

\begin{tabular}{|c|c|c|c|c|c|}
\hline \multirow[b]{2}{*}{ sample name } & \multicolumn{3}{|c|}{ Mixture composition (\%) } & \multirow[b]{2}{*}{$\begin{array}{c}\text { NMR } \\
\text { PO/CD ratio } \\
\end{array}$} & \multirow[b]{2}{*}{$\begin{array}{c}\text { Elemental analysis } \\
\text { PO/CD ratio }\end{array}$} \\
\hline & copolymer & $\beta-C D$ & $\mathrm{H}_{2} \mathrm{O}$ & & \\
\hline P0.25b1.75 & 0.25 & 1.75 & 98 & 2.35 & 1.89 \\
\hline $\mathrm{P} 0.75 \mathrm{~b} 1.25$ & 0.75 & 1.25 & 98 & 2.14 & 1.86 \\
\hline
\end{tabular}

Previous studies have shown that the average ratio of PO units threaded by cyclodextrins can vary between $2: 1$ (the limit based on size matching) and $3: 1^{22}$. Kinetic 
considerations must be taken into account as precipitation of the inclusion complexes can occur before the $\beta$-CD units can completely cover the PPO blocks.

The polypseudorotaxane structures of the precipitate complexes posses some degree of order. Thus, some characteristic peaks could be detected by XRD measurements. Figure 4 shows X-ray diffractograms for $10 \mathrm{R} 5 / \beta-\mathrm{CD}$ samples compared to a pure $\beta$-CD sample. The latter was obtained after applying the same treatment, i.e. freeze drying of its aqueous solution and a subsequent washing with THF. The diffraction patterns show some important differences. The most intense peak present in the complexed mixture at $17.6^{\circ}$ is characteristic of the channel type structure for $\beta$-cyclodextrin ${ }^{26}$. In these structures, the $\beta-\mathrm{CD}$ molecules are ordered head-to-head forming channels. For polyrotaxanes, the copolymer chains are threaded inside those channels. Our original procedure to prepare these mixtures consisted on mixing bulk Pluronics with an aqueous solution of $\mathrm{CD}$. This procedure could lead to highly heterogeneous structures because of the rapid macroscopic phase separation observed for these $\beta$-CD complexes. In order to ascertain whether this is a relevant fact, we have prepared the $\beta$-CD/reverse Pluronic inclusion complex in different ways: besides using different ratios for the mixture of the $\mathrm{CD}$ aqueous solution and bulk Pluronic, we have also prepared a sample mixing aqueous Pluronic with a CD solution. Moreover, an additional sample was obtained separating the precipitate by filtration (instead of recovering it by sedimentation as in our usual procedures). In all cases, the same product is obtained. As can be seen in Figure 4, a perfect match between their X-ray diffraction patterns is observed, confirming the remarkable stability of the IC produced. An additional experiment was performed using infrared spectroscopy (see Supporting Information, Figure S3) with the ICs obtained using different $\mathrm{CD}$ /Pluronic ratios. A perfect match is 
also observed for both solids using this technique, confirming the identical stoichiometry of the ICs.

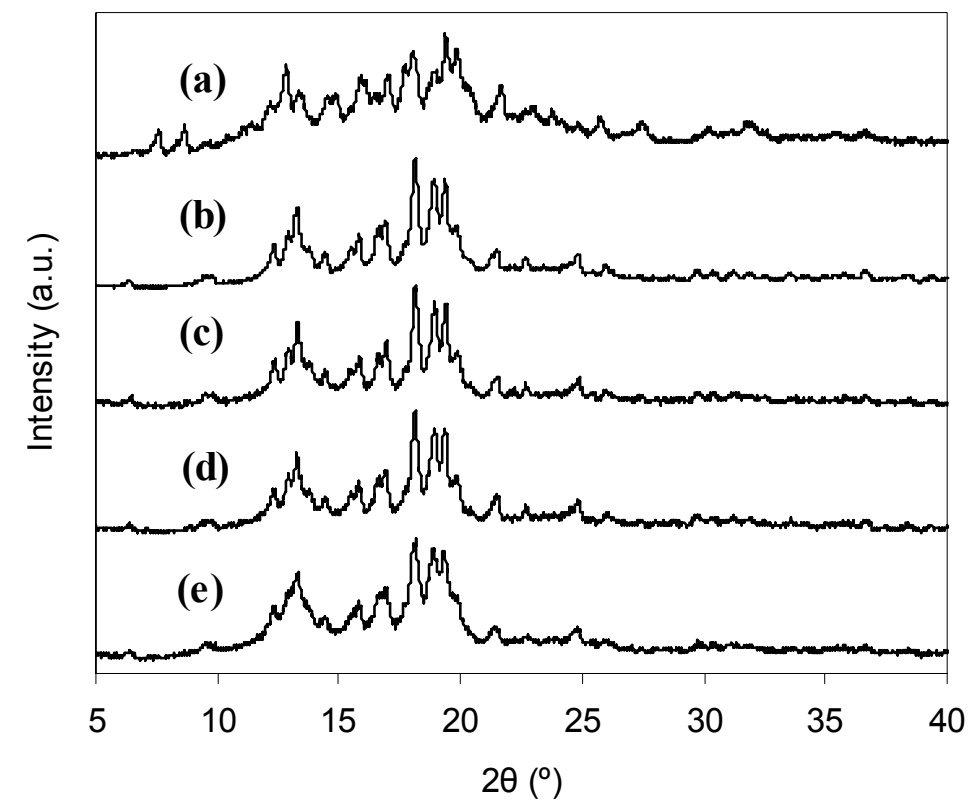

Figure 4. XRD patterns of: $\beta$-CD (a), P0.25b1.75 prepared by sedimentation (b), P0.25b1.75 prepared by filtration (c), P0.75b1.25 prepared by mixing a $\mathrm{CD}$ aqueous solution with pure Pluronic (d), P0.75b1.25 prepared by mixing a $2.5 \%$ CD solution with $1.8 \%$ aqueous Pluronic (e).

Complex formation of 10R5 with $\boldsymbol{\alpha}-\mathrm{CD}$. The supramolecular aggregates formed with $\alpha-C D$ and 10R5 are different from the complexes obtained with $\beta-C D$. As can be seen in Figure 2, once the polypseudorotaxane structures are formed, there is an additional hydrophobic interaction between PPO blocks. The higher solubility of $\alpha$-CD compared to that of $\beta-C D$ allows us to prepare aqueous solutions with higher concentrations of $\alpha-$ $\mathrm{CD}$ and the copolymer. When these $\alpha-\mathrm{CD}$ and 10R5 solutions are mixed, viscous white fluid gels can be obtained. Depending on the $\alpha-\mathrm{CD} / 10 \mathrm{R} 5$ ratio and on the total 
concentration, the kinetics of the complex formation process and the final aspect of the aggregates can vary leading to either "fluid" or "solid" gels (i.e. "weak gels" or "true gels", respectively, see Figure S4). Despite the fact that $\alpha$-CD molecules must surpass the PPO blocks to reach the PEO central blocks, the complexation is a very fast process, and in many cases it can be observed within minutes. Finally, stable gels are obtained which appear to be homogeneous. In this case, the mixtures were not washed to eliminate the solutes which are not forming part of inclusion complexes because we were interested in the gelation behaviour of these systems rather than in the IC structures themselves.

Figure 5 shows the sol-gel phase diagram for $10 \mathrm{R} 5 / \alpha-\mathrm{CD}$ aqueous mixtures using 10\% (w/w) (see also Figure S4 for other $\alpha-\mathrm{CD}$ concentrations). Pluronic 10R5 cannot form gels for $\alpha$-CD concentrations below $10 \%$ and copolymer concentrations below $25 \%$. The obtained gels do not flow when the vial is inverted, but when it is slightly shaken the gel starts to flow, i.e. they show a thixotropic behaviour. This is a standard procedure, frequently found in the literature, to classify sol-gel systems. We have observed that, in limit cases, the flow properties of a gel depend on the vial diameter and the volume of gel. For the same sample, different results were found when changing these parameters (see Supporting Information, Table S1).

It is noticeable that the $\alpha-\mathrm{CD} / 10 \mathrm{R} 5$ mole ratio of the limit mixture characterized as a gel is ca. one. This is quite a low value considering than up to 11 cyclodextrin moieties can fit complexing the central block of 10R5. This result suggests that gels are formed mainly by hydrophobic interactions between PPO blocks rather than by association between complexed CD/PEO blocks. Pluronic 10R5 can not form gels by itself, and $\alpha-C D$ is needed to trigger the gelation process. This observation can be corroborated studying the rheology of the obtained gels. 


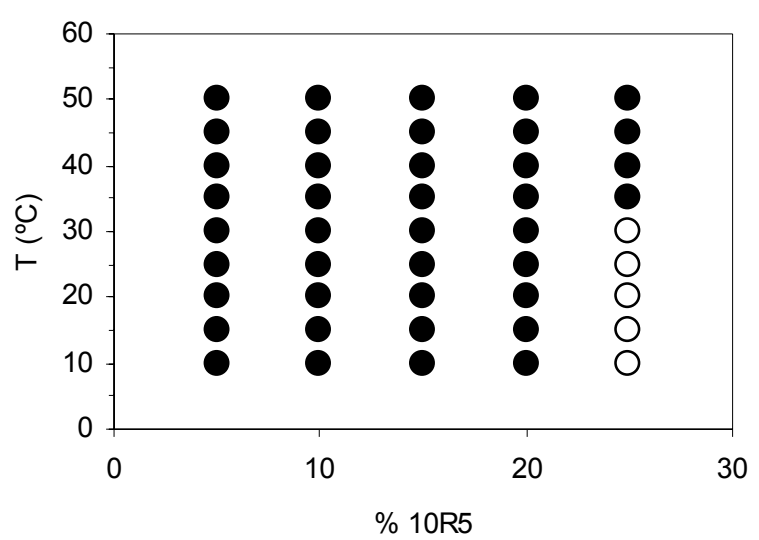

Figure 5. Temperature-concentration phase diagrams of aqueous Pluronic 10R5 in the presence of $10 \% \alpha$-CD. Sol phase $(\bullet)$ Gel phase (०)

Viscosity measurements were performed in order to characterize the rheological behaviour of these $10 \mathrm{R} 5 / \alpha$-CD fluid gels using samples containing $10 \%(\mathrm{w} / \mathrm{w})$ of $\alpha$-CD and different concentrations of 10R5. The viscosities of these mixtures as a function of the shear rate show a pseudoplastic (shear-thinning) behaviour (Figure 6). Besides, as in the case of direct Pluronics 5 , 10R5 gels exhibit a thixotropic behaviour. These thixotropic effects are not excessively important in the case of moderate shear rates for the short measuring periods studied. In the inset of Figure 6, a breakdown in the tendency of the viscosities (measured at $40 \mathrm{~s}^{-1}$ ) between 15 and $20 \%(\mathrm{w} / \mathrm{w})$ of $10 \mathrm{R} 5$ is clearly observed. This concentration corresponds to a ratio of one $\alpha$-CD moiety per 10R5 molecule. This corroborates our supposition that the intermolecular PPO-PPO interactions are the main responsible for the thickening of the aqueous mixtures. When $\alpha$-CD is added to the copolymer solutions, PEO blocks are complexed by threading one of these cyclodextrins, so the conformation of the poloxamer molecules changes facilitating the interactions between PPO blocks. $\alpha-C D / 10 R 5$ ratios of less than one $\alpha$ CD per 10R5 molecule (i.e. higher values of \% 10R5) correspond to mixtures with 
"free" poloxamer chains, whose disordered conformations contribute to an increase in the viscosity of the gel, as seen in the inset of Figure 6.

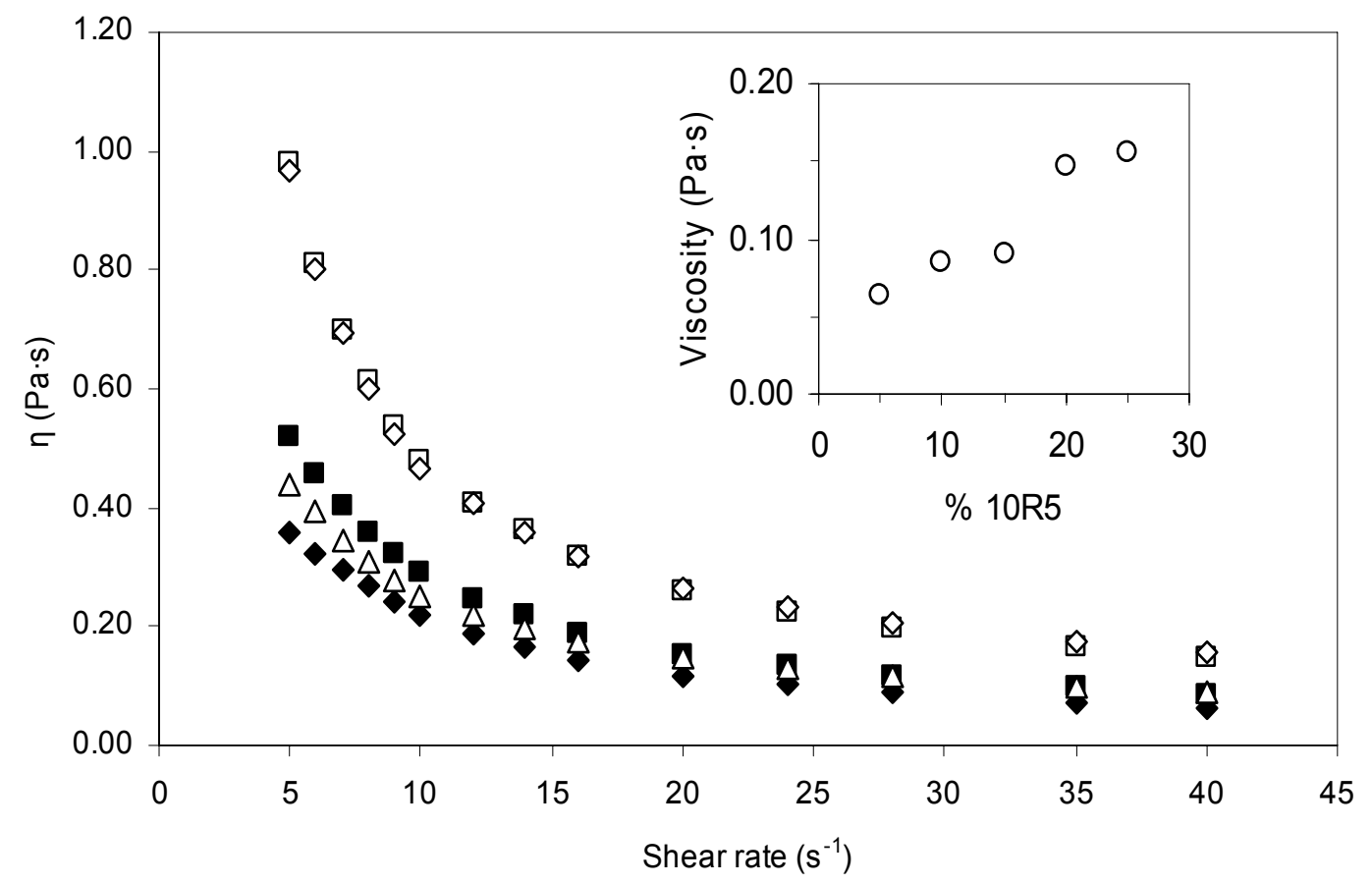

Figure 6. Viscosities of gels composed by $10 \%(w / w)$ of $\alpha-C D$ and different concentrations of 10R5: $5 \%(\diamond), 10 \%(\bullet), 15 \%(\Delta), 20 \%(\square)$, and $25 \%(\diamond)$ as a function of shear rate. The inset shows the dependence of the viscosity with the concentration of $10 \mathrm{R} 5$ measured at a constant shear rate of $40 \mathrm{~s}^{-1}$.

Direct poloxamers (PEO-PPO-PEO) previously studied in the literature form $^{5}$ gels with $\alpha-C D$ by a different mechanism: the gelation is primarily induced by the partial formation of the inclusion complexes of PEO segments with $\mathrm{CD}$ (with more than one CD moiety per block of PEO). It has to be noticed that, for direct poloxamers, the rotaxane structures are in the outer blocks. In contrast, the outer blocks of reverse Pluronics aggregate by hydrophobic interactions. Moreover, the self-assembling of the inner blocks via $\alpha-C D$ moieties is also necessary to obtain gels as can be deduced from the following experiment: when hydroxypropyl- $\alpha-C D$ is used, the aqueous mixture 
remains liquid, and no turbidity is observed. In this case, the $\alpha-C D$ derivative, which can also thread the poloxamer molecules, cannot form stable pseudorotaxane aggregations. A similar effect has been found in the case of $\beta-C D$ derivatives that do not form crystalline complexes with $\mathrm{PPO}^{14}$. We have also checked that mixtures of reverse Pluronic 10R5 and hydroxypropyl- $\beta-\mathrm{CD}$ remain liquid: neither precipitates nor turbidity were observed. Moreover, using equivalent amounts of glucose instead of CD yielded also clear solutions (see Supporting Information, Figures S5 and S6).

Figure 7 shows a comparison between XRD patterns for two different 10R5/ $\alpha$ $\mathrm{CD}$ gels and the complex formed between $\alpha-\mathrm{CD}$ and pure PEO $\left(\mathrm{M}_{\mathrm{w}}=400 \mathrm{~g} / \mathrm{mol}\right)$. The latter yields a white precipitate corresponding to a polyrotaxane with a channel type structure for $\alpha-C^{20}$. The characteristic XRD peak of $\alpha-C D$ channel structures (210) is located at about $20^{\circ}$, and 10R5 gels show this peak in their XRD patterns ${ }^{27}$. This result proves that, despite their gel character, these mixtures posses a partial crystalline order due to the PEO complexed groups. These groups tend to associate into ordered domains.

In the gels formed with $25 \%(\mathrm{w} / \mathrm{w})$ of copolymer and $10 \%(\mathrm{w} / \mathrm{w})$ of $\alpha-\mathrm{CD}$ there is less than one CD molecule per PEO block. Nevertheless, the XRD channel peak characteristic of the hexagonal packing (210) is clearly observed. The diffractograms for both $\mathrm{CD} / 10 \mathrm{R} 5$ mixtures show the same characteristic peak but the gels formed with $25 \%$ of copolymer present a larger amorphous domain (producing a curved baseline) that corresponds to disordered poloxamer chains with no cyclodextrins threading them. 


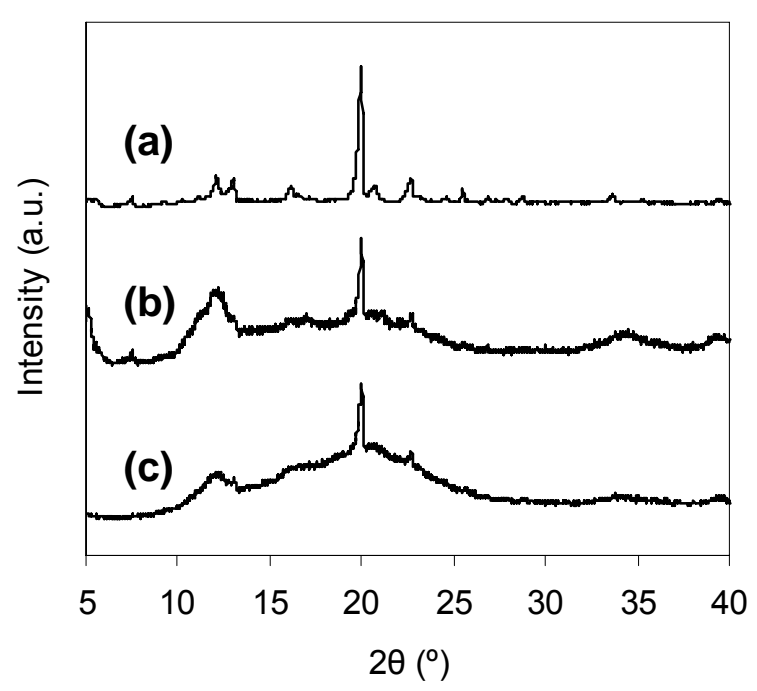

Figure 7. Wide-angle X-ray diffractograms for: $\alpha$-CD-PEO $\left(\mathrm{M}_{\mathrm{w}}=400 \mathrm{~g} / \mathrm{mol}\right)$ complex (a), $\alpha-\mathrm{CD} / 10 \mathrm{R} 5$ hydrogels containing $10 \%(\mathrm{w} / \mathrm{w})$ of $\alpha-\mathrm{CD}$ and $10 \%$ (b), or $25 \%$ (c) of $10 \mathrm{R} 5$.

The glass transitions temperatures $\left(\mathrm{T}_{\mathrm{g}}\right)$ of the poloxamer chains can be useful to ascertain their complexation state, which modifies the mobility of chain segments ${ }^{5}$. Data corresponding to different complexes formed between 10R5 and $\alpha-\mathrm{CD}$ are collected in Table 2. As can be seen, the glass transition temperatures of complexes with high $\mathrm{EO} / \mathrm{CD}$ ratios are practically equal to that of pure 10R5. For EO/CD mole ratios lower than two (i.e. 10R5/ $\alpha$-CD weight ratio lower than 0.18), the samples do not present a glass transition. The pseudorotaxane structure is completely filled when there is one CD per two EO units. When this occurs, the complexed PEO blocks tend to aggregate forming crystalline polyrotaxane structures instead of gels. On the other hand, if the $\mathrm{EO} / \mathrm{CD}$ ratio is higher than two, there are not enough $\alpha-\mathrm{CD}$ moieties to form complexes with all the PEO blocks, so the hydrophobic interactions between PPO blocks play a more important role. 
Table 2. Glass transition temperatures for $10 \mathrm{R} 5 / \alpha-\mathrm{CD}$ complexes

\begin{tabular}{|c|c|c|c|c|c|}
\hline \multirow[b]{2}{*}{ sample name } & \multicolumn{3}{|c|}{ mixture composition (\%) } & \multirow{2}{*}{$\begin{array}{c}\mathrm{EO} / \mathrm{CD} \\
(\mathrm{mol} / \mathrm{mol})\end{array}$} & \multirow[b]{2}{*}{$\mathrm{T}_{\mathrm{g}} /{ }^{\circ} \mathrm{C}$} \\
\hline & $10 \mathrm{R} 5$ & $\alpha-\mathrm{CD}$ & $\mathrm{H}_{2} \mathrm{O}$ & & \\
\hline$\alpha-C D$ & - & - & - & - & - \\
\hline $\mathrm{P} 0.25 \mathrm{a} 10$ & 0.25 & 10.00 & 89.75 & 0.28 & - \\
\hline P0.6a10 & 0.60 & 10.00 & 89.40 & 0.69 & - \\
\hline P1a10 & 1.00 & 10.00 & 89.00 & 1.10 & - \\
\hline P1.1a10 & 1.10 & 10.00 & 88.90 & 1.21 & - \\
\hline P3a10 & 3.00 & 10.00 & 87.00 & 3.34 & -64.8 \\
\hline P6a10 & 6.00 & 10.00 & 84.00 & 6.70 & -64.1 \\
\hline 10R5 & - & - & - & - & -65.2 \\
\hline
\end{tabular}

\section{Concluding remarks}

In contrast to the gelation behaviour observed for direct poloxamers ${ }^{28-30}$, Pluronic $10 \mathrm{R} 5$ can not gelify by itself. Nevertheless, when combined with $\alpha-C D$, this reverse Pluronic yields fluid gels. This effect is not observed when 10R5 is mixed with $\beta$-CD. These mixtures, formed after the complexation of the reverse poloxamer PPO blocks by the $\beta$ CD molecules, yield white precipitates with a polypseudorotaxane type structure and a certain crystalline order. It has to be noticed that these complexes need to be prepared using high amounts of water due to the lower solubility of $\beta$-CD compared to that of $\alpha$ CD (ca. 10-fold).

The higher water solubility of $\alpha-\mathrm{CD}$ allows us to prepare mixtures with higher amounts of both 10R5 poloxamer and cyclodextrin. The inclusion complexes between the inner PEO blocks of 10R5 and $\alpha$-CD lead to the formation of fluid gels whose viscosity can be tuned by varying the $10 \mathrm{R} 5 / \mathrm{CD}$ ratio. A certain amount of $\alpha-\mathrm{CD}$ is needed to form gels with this reverse poloxamer, but more viscous mixtures are obtained for low $\alpha-\mathrm{CD} / 10 \mathrm{R} 5$ ratios. This result suggests that, in the case of reverse Pluronics, the interactions responsible for the observed thickening are the hydrophobic ones between the PPO blocks. 


\section{ACKNOWLEDGEMENTS}

The authors acknowledge the financial aid from the Ministerio de Ciencia e Innovación (project MAT2007-65752). E.L. thanks for a Gobierno de Navarra grant (Plan de Formación y de I+D). We are also grateful to G. Tardajos (Universidad Complutense) for her help with the NMR results and to C. Cesteros (Universidad del País Vasco UPV/EHU) for DSC measurements.

\section{SUPPORTING INFORMATION}

Figures S1-S6. Table S1. This information is available free of charge via the Internet at http://pubs.acs.org

\section{REFERENCES}

(1) Van Tomme, S.; Storm, G.; Hennink, W. In Situ Gelling Hydrogels for Pharmaceutical and Biomedical Applications. Int. J. Pharm. 2008, 355, 1-18.

(2) Li, J.; Loh, X. Cyclodextrin-Based Supramolecular Architectures: Syntheses, Structures, and Applications for Drug and Gene Delivery. Adv. Drug Deliv. Rev. 2008, 60, 1000-1017.

(3) Li, J.; Li, X.; Ni, X.; Wang, X.; Li, H.; Leong, K. Self-Assembled Supramolecular Hydrogels Formed by Biodegradable PEO-PHB-PEO Triblock Copolymers and Alpha-Cyclodextrin for Controlled Drug Delivery. Biomaterials 2006, 27, 41324140.

(4) Nielsen, A.; Steffensen, K.; Larsen, K. Self-Assembling Microparticles with Controllable Disruption Properties Based on Cyclodextrin Interactions. Colloids and surfaces. B, Biointerfaces 2009, 73, 267-275.

(5) Ni, X.; Cheng, A.; Li, J. Supramolecular Hydrogels Based on Self-Assembly between PEO-PPO-PEO Triblock Copolymers and Alpha-Cyclodextrin. Journal of biomedical materials research.Part A 2009, 88A, 1031-1036. 
(6) van de Manakker, F.; Vermonden, T.; van Nostrum, C.; Hennink, W. SelfAssembling Hydrogels Based on Beta-cyclodextrin/cholesterol Inclusion Complexes. Macromolecules 2008, 41, 1766-1773.

(7) Koopmans, C.; Ritter, H. Formation of Physical Hydrogels Via Host-Guest Interactions of Beta-Cyclodextrin Polymers and Copolymers Bearing Adamantyl Groups. Macromolecules 2008, 41, 7418-7422.

(8) Wintgens, V.; Daoud Mahammed, S.; Gref, R.; Bouteiller, L.; Amiel, C. Aqueous Polysaccharide Associations Mediated by Beta-Cyclodextrin Polymers. Biomacromolecules 2008, 9, 1434-1442.

(9) Dodziuk, H. In Cyclodextrins and their complexes: chemistry, analytical methods, applications; Wiley-VCH: 2006; .

(10) Li, J.; Ni, X.; Leong, K. Injectable Drug-Delivery Systems Based on Supramolecular Hydrogels Formed by Poly(Ethylene Oxide) and AlphaCyclodextrin. J. Biomed. Mater. Res. 2003, 65A, 196-202.

(11) Harada, A. Polyrotaxanes. Acta polymerica 1998, 49, 3-17.

(12) Harada, A.; Takashima, Y.; Yamaguchi, H. Cyclodextrin-Based Supramolecular Polymers. Chem. Soc. Rev. 2009, 38, 875-882.

(13) Li, J.; Li, X.; Toh, K.; Ni, X.; Zhou, Z.; Leong, K. Inclusion Complexation and Formation of Polypseudorotaxanes between Poly[(Ethylene Oxide)-Ran(Propylene Oxide)] and Cyclodextrins. Macromolecules 2001, 34, 8829-8831.

(14) Harada, A.; Okada, M.; Li, J.; Kamachi, M. Preparation and Characterization of Inclusion Complexes of Inclusion Complexes of Poly(Propylene Glycol) with Cyclodextrins. Macromolecules 1995, 28, 8406-8411.

(15) Harada, A.; LI, J.; Kamachi, M. Synthesis of a Tubular Polymer from Threaded Cyclodextrins. Nature 1993, 364, 516-518.

(16) Harada, A.; Li, J.; Nakamitsu, T.; Kamachi, M. Preparation and Characterization of Polyrotaxanes Containing Many Threaded Alpha-Cyclodextrins. J. Org. Chem. 1993, 58, 7524-7528.

(17) Harada, A.; Li, J.; Kamachi, M. The Molecular Necklace - a Rotaxane Containing Many Threaded Alpha-Cyclodextrin. Nature 1992, 356, 325-327.

(18) Alexandridis, P.; Hatton, T. Poly(Ethylene Oxide)-Poly(Propylene Oxide)Poly(Ethylene Oxide) Block Copolymer Surfactants in Aqueous Solutions and at Interfaces: Thermodynamics, Structure, Dynamics, and Modeling. Colloids and surfaces.A, Physicochemical and engineering aspects 1995, 96, 1-46.

(19) Trong, L.; Djabourov, M.; Ponton, A. Mechanisms of Micellization and Rheology of PEO-PPO-PEO Triblock Copolymers with various Architectures. J. Colloid Interface Sci. 2008, 328, 278-287. 
(20) Li, J.; Ni, X.; Zhou, Z.; Leong, K. Preparation and Characterization of Polypseudorotaxanes Based on Block-Selected Inclusion Complexation between Poly(Propylene Oxide)-Poly(Ethylene Oxide)-Poly(Propylene Oxide) Triblock Copolymers and Alpha-Cyclodextrin. J. Am. Chem. Soc. 2003, 125, 1788-1795.

(21) Tsai, C.; Leng, S.; Jeong, K.; Van Horn, R.; Wang, C. Supramolecular Structure of Beta-Cyclodextrin and Poly(Ethylene Oxide)-Block-Poly(Propylene Oxide)-BlockPoly(Ethylene Oxide) Inclusion Complexes. Macromolecules 2010, 43, 9454-9461.

(22) Tsai, C.; Zhang, W.; Wang, C.; Van Horn, R.; Graham, M.; Huang, J.; Chen, Y.; Guo, M. Evidence of Formation of Site-Selective Inclusion Complexation between Beta-Cyclodextrin and Poly(Ethylene Oxide)-Block-Poly(Propylene Oxide)Block-Poly(Ethylene Oxide) Copolymers. J. Chem. Phys. 2010, 132, 204903.

(23) Huang, J.; Zhou, Z.; Wei, M.; Chen, Y.; Chang, P. Soy Protein-Based Nanocomposites Reinforced by Supramolecular Nanoplatelets Assembled from Pluronic polymers/beta-Cyclodextrin Pseudopolyrotaxanes. J Appl Polym Sci 2007, 107, 409-417.

(24) Perry, C.; Hebraud, P.; Gernigon, V.; Brochon, C.; Lapp, A. Pluronic and BetaCyclodextrin in Water: From Swollen Micelles to Self-Assembled Crystalline Platelets. Soft matter 2011, 7, 3502-3512.

(25) Yang, C.; Ni, X.; Li, J. Synthesis of Polyrotaxanes Consisting of Multiple AlphaCyclodextrin Rings Threaded on Reverse Pluronic PPO-PEO-PPO Triblock Copolymers Based on Block-Selected Inclusion Complexation. European polymer journal 2009, 45, 1570-1579.

(26) Okumura, H.; Kawaguchi, Y.; Harada, A. Preparation and Characterization of Inclusion Complexes of Poly(Dimethylsiloxane)s with Cyclodextrins. Macromolecules 2001, 34, 6338-6343.

(27) Chung, J.; Kang, T.; Kwak, S. Guest-Free Self-Assembly of Alpha-Cyclodextrins Leading to Channel-Type Nanofibrils as Mesoporous Framework. Langmuir 2007, 23, 12366-12370.

(28) Chaibundit Micellization and Gelation of Mixed Copolymers P123 and F127 in Aqueous Solution. Langmuir 2007, 23, 9229-9236.

(29) Antunes, F.; Gentile, L.; Rossi, C.; Tavano, L.; Ranieri, G. Gels of Pluronic F127 and Nonionic Surfactants from Rheological Characterization to Controlled Drug Permeation. Colloids and surfaces.B, Biointerfaces 2011, 87, 42-48.

(30) Chaibundit, C. Effect of Ethanol on the Micellization and Gelation of Pluronic P123. Langmuir 2008, 24, 12260-12266. 


\section{Supporting Information}

Title: Self-assembled Supramolecular Gels of Reverse Poloxamers and Cyclodextrins

Authors: E. Larrañeta, J.R. Isasi
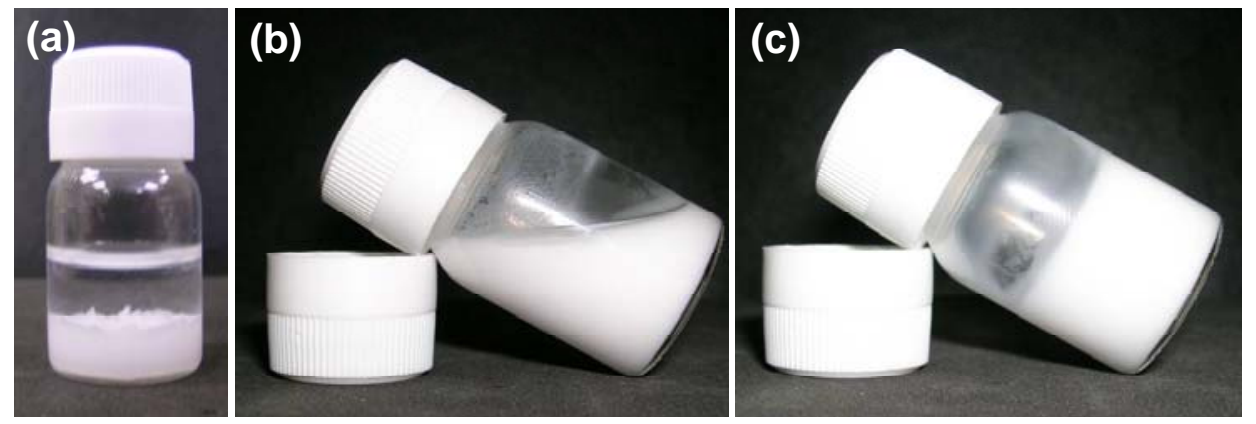

Figure S1. Photographs of different 10R5 complexes: 10R5/ $\beta$-CD complex (a), $10 \mathrm{R} 5 / \alpha-\mathrm{CD}$ weak gel (b) and 10R5/ $\alpha-\mathrm{CD}$ true gel (c).

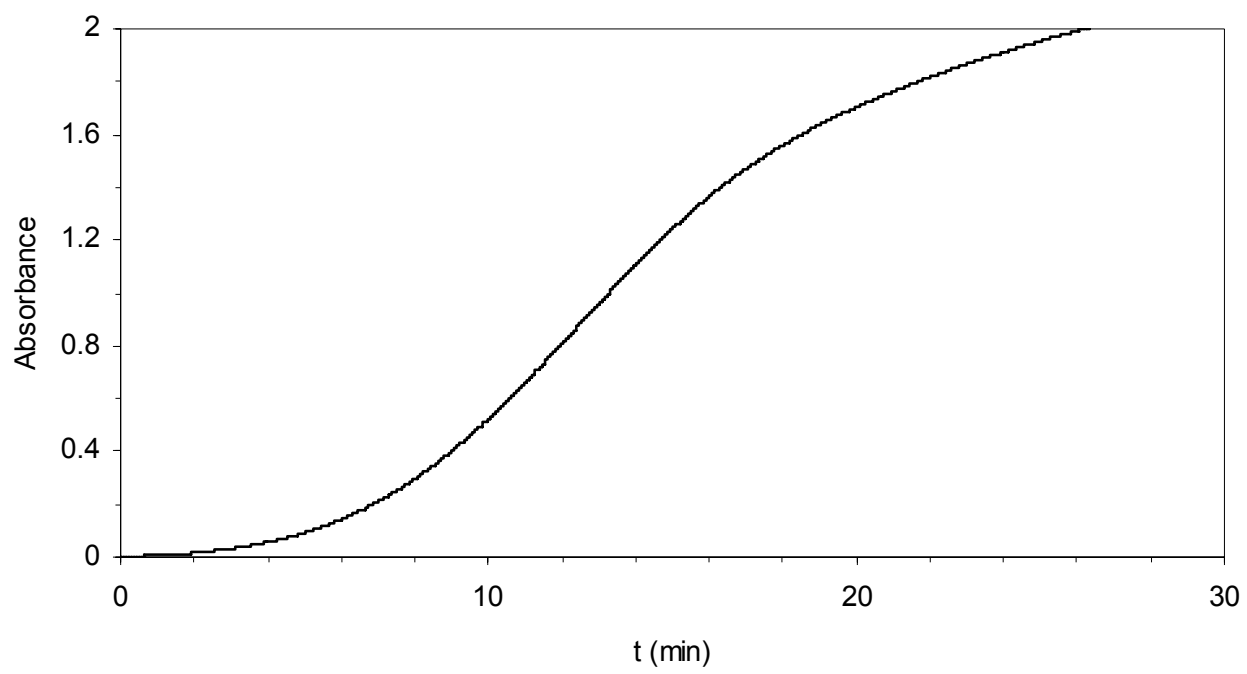

Figure S2. Turbidity of a mixture containing $0.5 \%$ of Pluronic $10 \mathrm{R} 5$ and $1.5 \%$ of $\beta$-CD measured by UV-vis (JASCO V-630) at $600 \mathrm{~nm}$ as a function of time. 


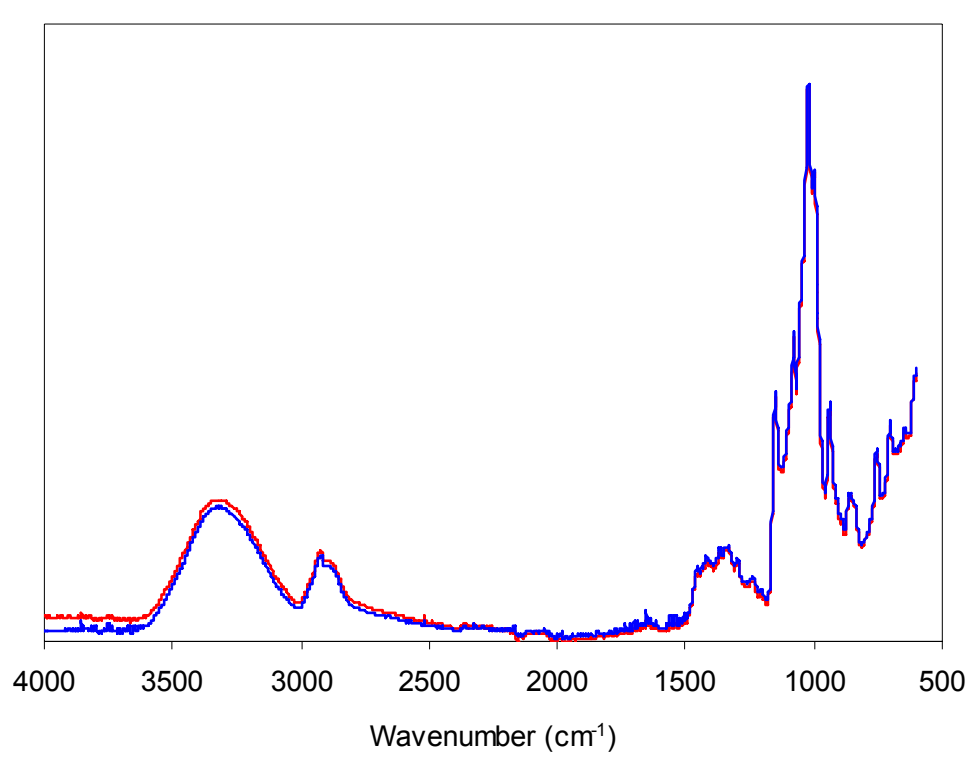

Figure S3. FTIR spectra of P0.25b1.75 (red) and P0.75b1.25 (blue) complexes prepared by filtration.
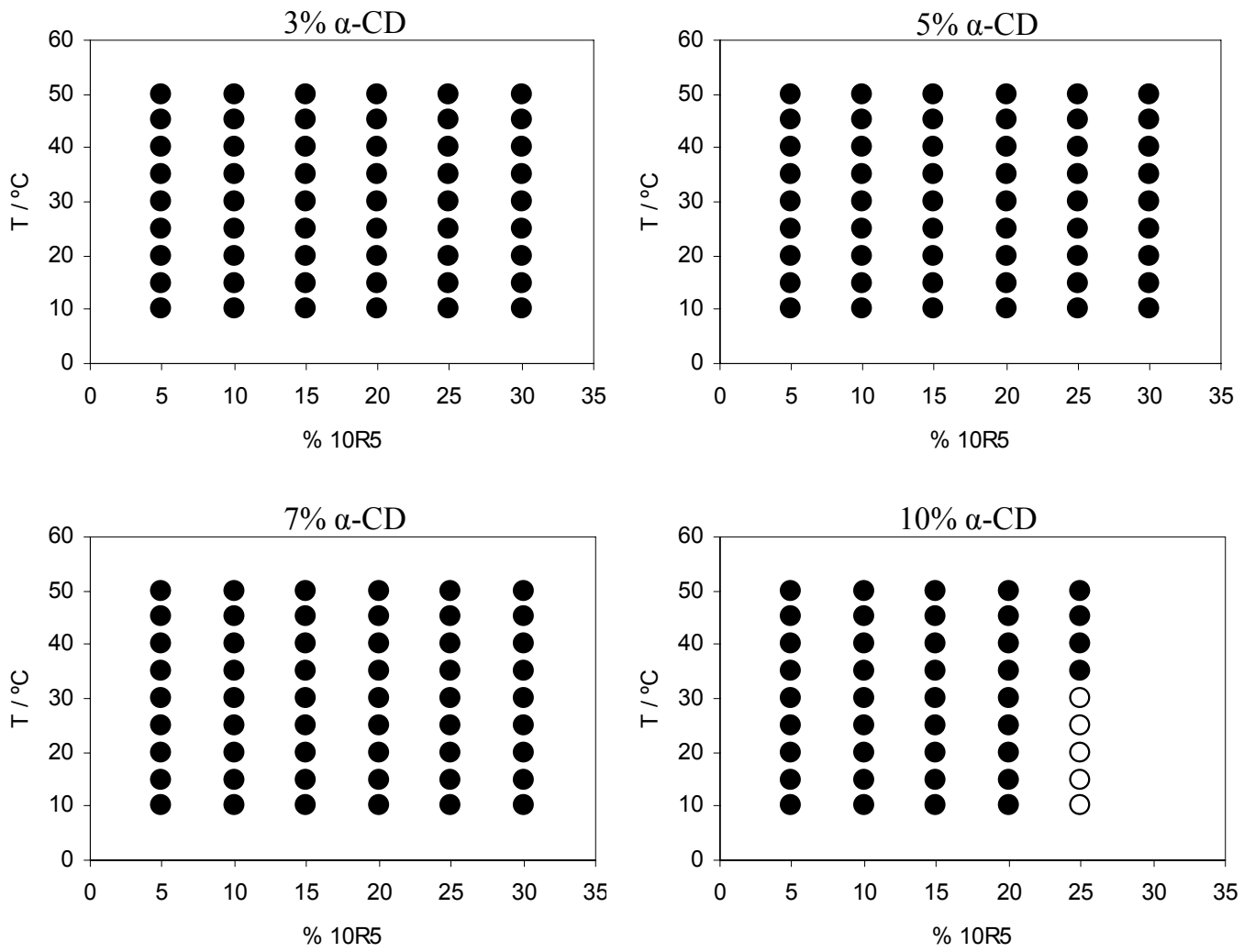

Figure S4. Temperature-concentration phase diagrams of aqueous Pluronic 10R5 in the presence of $\alpha$-CD. Sol phase $(\bullet)$ Gel phase $(\circ)$ 
Table S1. Rheological behaviour as a function of the flask diameter and the amount of gel for different P25a10 mixtures.

\begin{tabular}{cccc}
\hline Amount of gel $(\mathrm{g})$ & \multicolumn{3}{c}{ Flask diameter $(\mathrm{mm})$} \\
\cline { 2 - 4 } & 23.0 & 30.5 & 38.5 \\
\hline 5 & Does not flow & Does not flow & Flows \\
10 & Flows & Flows & Flows \\
\hline
\end{tabular}

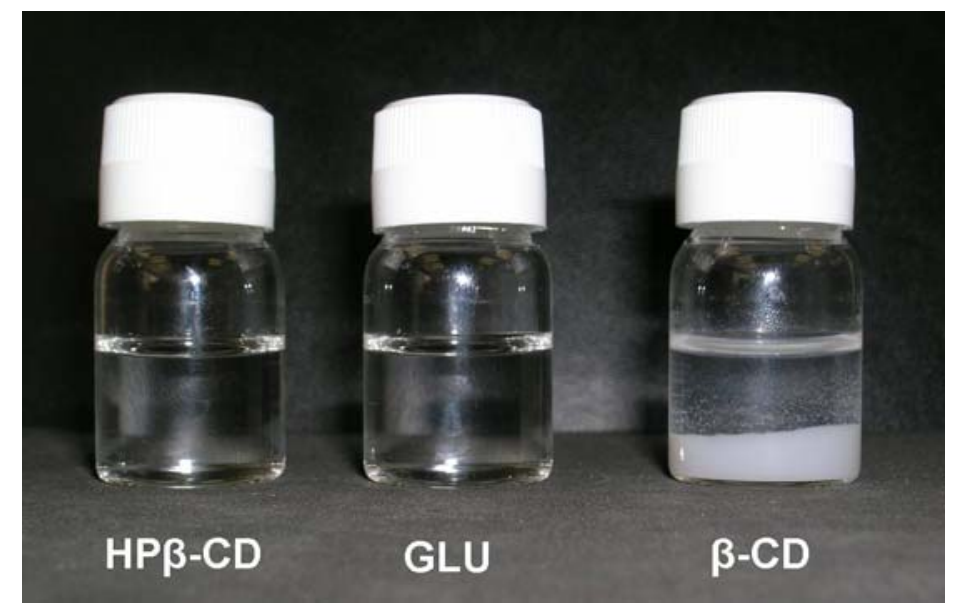

Figure S5. Aqueous mixtures of Pluronic $10 \mathrm{R} 5$ and $\mathrm{HP} \beta-\mathrm{CD}$, glucose, and $\beta-\mathrm{CD}$ using the same ratios for the components of the mixtures.

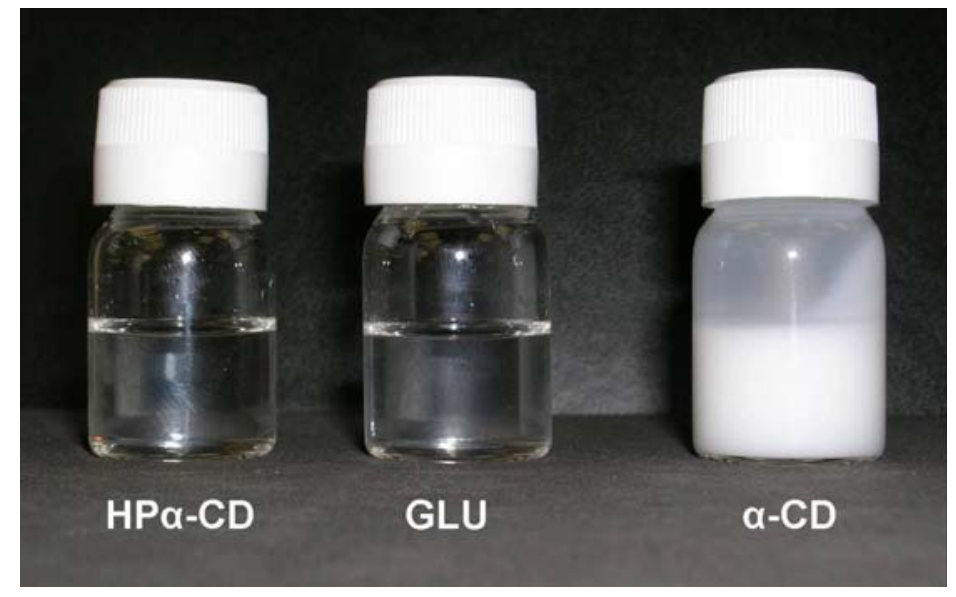

Figure S6. Aqueous mixtures of Pluronic 10R5 and HP $\alpha-C D$, glucose, and $\alpha-C D$ using the same ratios for the components of the mixtures. 